\title{
BEAM COLLIMATOR DESIGN FOR THE 3GEV SYNCHROTRON OF THE JAERI-KEK JOINT PROJECT
}

\author{
K. Yamamoto, M. Kinsho, F. Noda, JAERI \\ Y. Irie, M. Shirakata, KEK
}

\begin{abstract}
In order to localize the beam loss on the restricted area, the beam collimation system design has been performed for the $3 \mathrm{GeV}$ synchrotron of JAERI-KEK joint project. The beam loss distribution has been calculated with Monte-Carlo simulation code STRUCT developed at Fermi national laboratory. The collimation efficiency was calculated for two operation tune, where more than 98\% of traced beam loss is lost around the ring for collimation system for each operation tune.
\end{abstract}

\section{INTRODUCTION}

Japan Atomic Energy Research Institute (JAERI) and High Energy Accelerator Research Organization (KEK) have been going ahead with the joint project of high intensity proton accelerators. The accelerator complex consists of a $600 \mathrm{MeV}$ linac, a $3 \mathrm{GeV}$ rapid cycling synchrotron, and a $50 \mathrm{GeV}$ synchrotron ring [1].

The $3 \mathrm{GeV}$ synchrotron ring accelerates a proton beam from the $400-600 \mathrm{MeV}$ Proton Linac up to $3 \mathrm{GeV}$ and supplies it to the $50 \mathrm{GeV}$ synchrotron ring. The $3 \mathrm{GeV}$ synchrotron ring also supplies the neutron production target with the $3 \mathrm{GeV}$ proton beam in order to produce a high intensity neutron beam. For this purpose, the $3 \mathrm{GeV}$ synchrotron ring aims to generate a high power beam of 1MW by setting $25 \mathrm{~Hz}$ as the repetition rate of acceleration and $8.3 \times 10^{13}$ as the proton number per acceleration [2].

From experience of the accelerator operation, the average beam loss should be kept at an order of 1 watt per meter for hands-on maintenance [3] [4]. Since it is very difficult to control the beam loss at such a low level, the only measure we can take is to localize any of the losses in a restricted area, where deliberate modules should be provided for quick coupling and remote handling in order to mitigate the personal doses. Accordingly, we have designed the beam collimation system for the purpose of the beam loss localization.

\section{COLLIMATOR SYSTEM}

The classical two-stage collimation system is applied to our case [5]. In this system, halo particles are scattered by the primary tungsten collimators, and the secondly copper collimators absorb those particles. Three types of primary collimators are prepared in this system: the one is off-momentum particle (longitudinal) collimator, and the others are horizontal and vertical (transverse) halo collimators. Transverse primary collimators are set in the second half of injection straight section, and longitudinal primary collimator is located near the dispersion maximum point above transverse primary collimators.
The thickness of primary tungsten collimator is $3 \mathrm{~mm}$ for transverse collimation and is $0.01 \mathrm{~mm}$ for longitudinal collimation, respectively. These values were decided from scattering angle of particles. Particle which was scattered by longitudinal collimator is diffused between collimator aperture and physical aperture, and pass through the vacuum pipe until it hits transverse collimators again. Five secondary collimators are set downstream of the transverse collimators, and its thickness is set to be $200 \mathrm{~mm}$ by reason of necessity to stop several hundred $\mathrm{MeV}$ proton. Every primary collimator restricts apertures to $216 \pi \mathrm{mm}$.mrad emittance and $1 \%$ momentum dispersion. All secondary collimators restrict apertures to $256 \pi \mathrm{mm}$.mrad emittance. (Transverse primary collimators and all of secondary collimators are set in the dispersion-free straight section.). All of the other vacuum pipes are designed $247 \mathrm{~mm}$ in diameter except for the dipole magnet (BM). The diameter of vacuum pipe in the BM is set up at $177 \mathrm{~mm}$, and both values are larger than the beam size of $324 \pi \mathrm{mm}$.mrad emittance and $1 \%$ momentum dispersion. Location of collimator system and twiss parameters are shown in Fig.1.

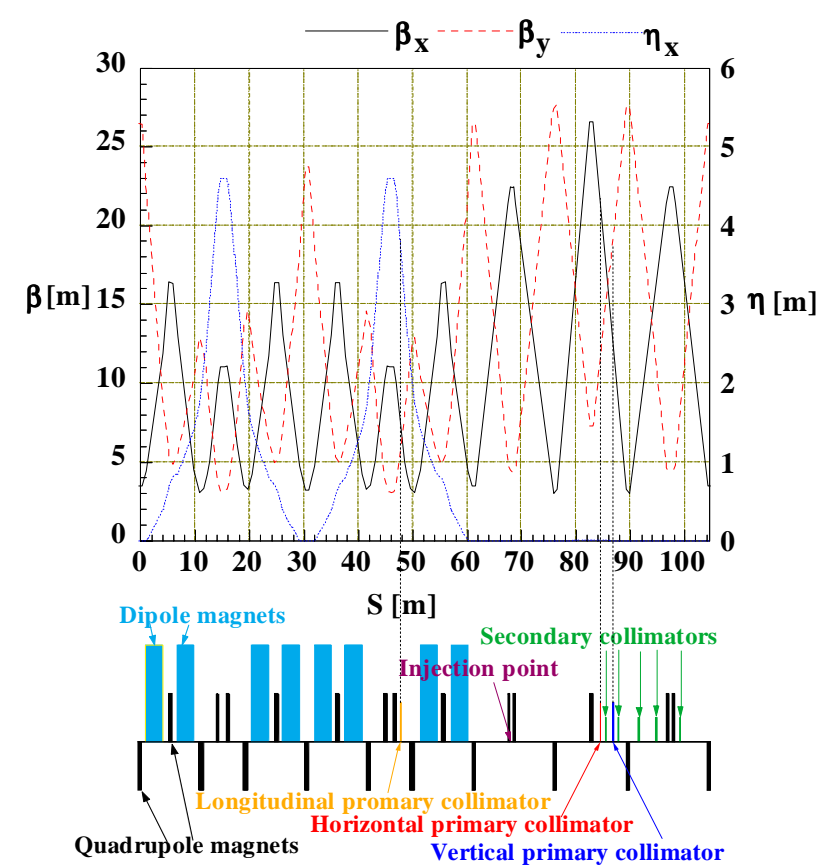

Fig.1 Location of collimator system and twiss parameters of one superperiod. 


\section{CALCULATION}

The beam loss distribution was estimated by STRUCT code. STRUCT was developed in FNAL. It calculates beam track and scattering effect. In these calculations, I assumed two types of particle distributions in the transverse and longitudinal phase space. The one is transverse halo distribution, and the other is off momentum halo particle distribution. Transverse halo is distributed from $216 \pi \mathrm{mm} . \mathrm{mrad}$ to $246 \pi \mathrm{mm} . \mathrm{mrad}$ emittance transversely and longitudinally distributed from $-1 \%$ to $1 \%$ momentum within the RF bucket shape. Longitudinal off momentum particles are set from $1 \%$ to $1.5 \%$ momentum longitudinally and distributed within $216 \pi \mathrm{mm}$.mrad emittance transversely. In the case of transverse halo, beam with large horizontal emittance, has small emittance vertically and vice versa. This represents anti-correlated painting. These particle distributions are shown in Fig.2. All calculations are assumed that the amount of total beam loss was $4 \mathrm{~kW}$.
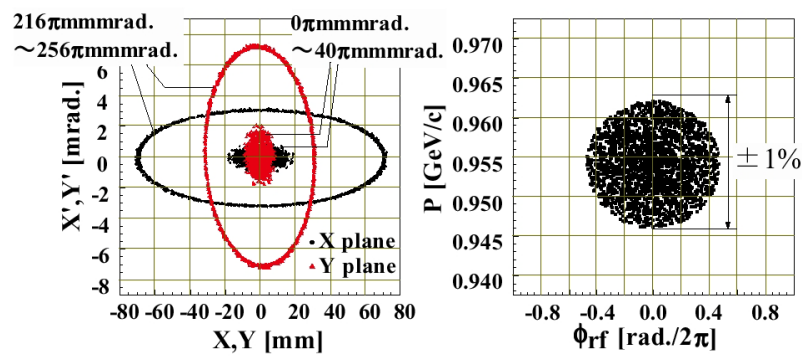

Fig.2 Initial particle distribution of transverse halo Transverse phase plane (left) and longitudinal phase plane (right).

\section{RESULTS AND DISCUSSION}

\subsection{Tune dependence}

Tune shift due to space charge is an ineluctable problem for these high intensity machines. We shall choose least tune shift (and least beam loss) operation point.

Therefore, in the first place, we investigated the tune dependence of collimation system with two operation points, $\left(v_{x}, v_{y}\right)=(7.35,5.8)$ and $(6.7,5.7)$. Phase advances from primary collimators to secondary collimators are different those for two operations. Those values are listed in Table 1. And the calculation results are shown in Fig.3. In both case, beam losses of transverse halo are well localized by these collimation system. The collimation efficiency, which is defined by the ratio of the amount of
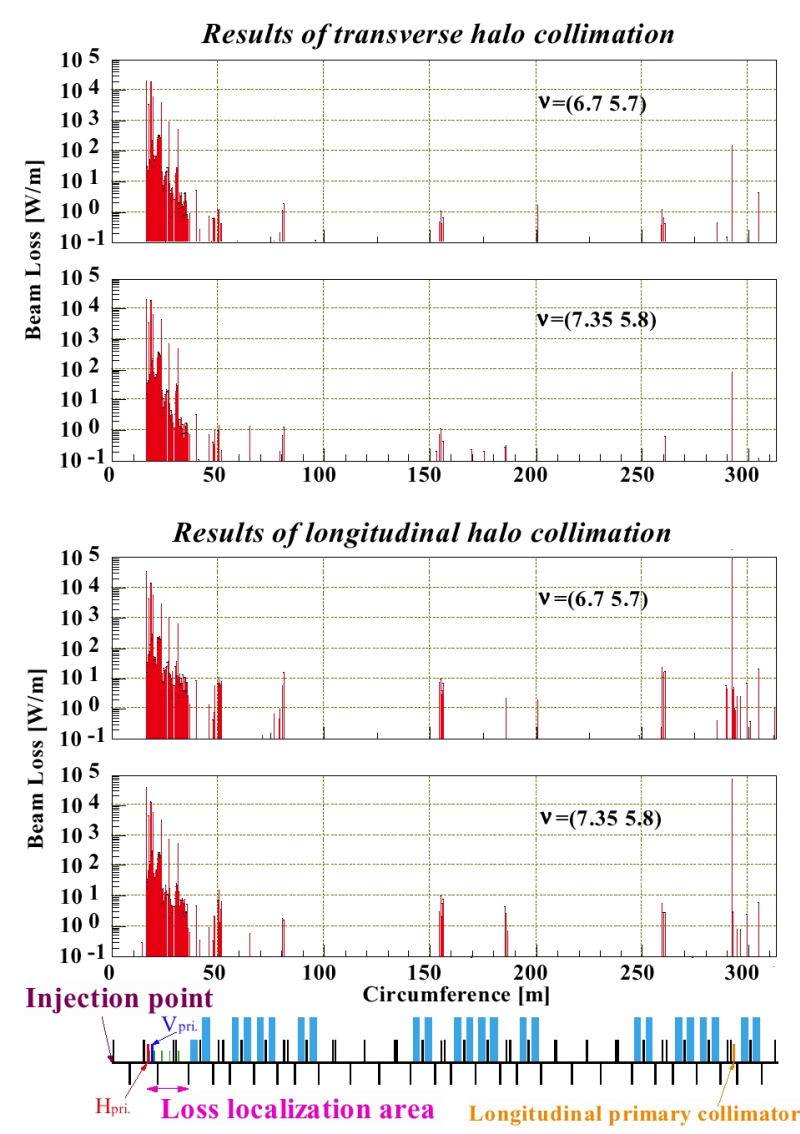

Fig.3. Beam loss distributions along the ring with different operations. Above two histograms represent the results of the collimation of transverse halo. Below two histograms are the results of the collimation of off momentum particles. Total beam loss was assumed $4 \mathrm{~kW}$ in these calculations.

lost beam in the loss locarization area (see Fig.3) to the amount of lost beam in the whole ring, is $99.4 \%$ at $v_{x}=7.35$ operation and $98.9 \%$ at $v_{x}=6.7$ operation, respectivery. But the collimation efficiencies of off momentum particles are different between both operation points (97.6\% at $v_{x}=7.35$ and $94.4 \%$ at $\left.v_{x}=6.7\right)$. It seems that these results are attributed to the difference of regional $\beta$ and $\eta$ dependence on momentum rather than the difference of tune, because the results of transverse halo collimations at both operation points are not so different as the results of longitudinal collimations. The momentum distributions of transverse halo are smaller than that of off momentum particles.

Table 1 Phase advance of two operation points.

\begin{tabular}{|c|c|c|c|c|c|c|}
\hline & \multicolumn{5}{|c|}{ collimator 1 collimator 2 collimator 3 collimator 4 collimator 5} \\
\hline \multirow{2}{*}{$\begin{array}{l}\text { Phase advance [degree] } \\
\left(v_{x}, v_{y}\right)=(7.35,5.8)\end{array}$} & Horizontal & 4.49 & 19.67 & 79.19 & 102.08 & 115.34 \\
\hline & Vertical & - & 13.11 & 22.32 & 38.08 & 85.56 \\
\hline \multirow{2}{*}{$\begin{array}{l}\text { Phase advance [degree] } \\
\left(v_{x}, v_{v}\right)=(6.7,5.7)\end{array}$} & Horizontal & 5.15 & 20.07 & 62.05 & 80.07 & 92.67 \\
\hline & Vertical & - & 14.39 & 24.45 & 39.41 & 75.91 \\
\hline
\end{tabular}




\subsection{Physical aperture}

For the effective collimation, it is very important to secure enough physical aperture size. Our designs of physical apertures are $177 \mathrm{~mm}$ at $\mathrm{BM}$ and $247 \mathrm{~mm}$ at others. These values are decided by gap height of BM and bore diameter of Q-magnets. Secondary we investigated necessary physical aperture size for the effective collimation. In these calculations, we use the operation point $\left(v_{x}, v_{y}\right)=(7.35,5.8)$, and physical apertures are changed from $70 \%$ to $130 \%$ of the designed sizes. Figure 4 shows the collimation efficiency with those physical apertures.

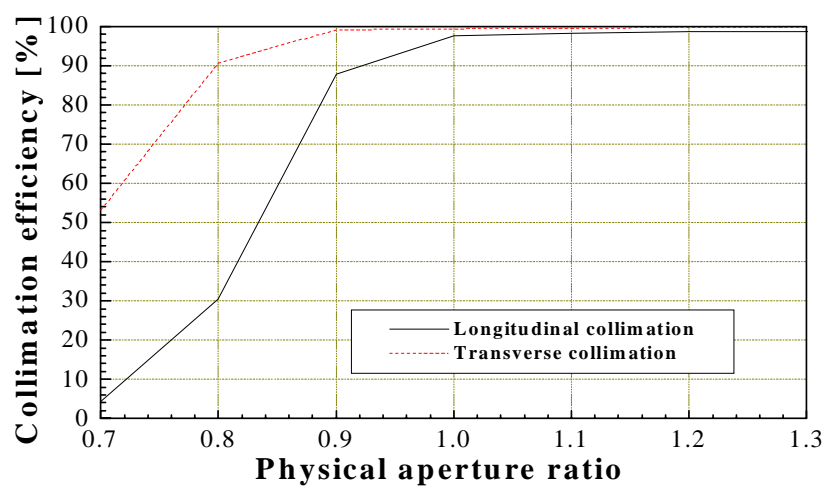

Fig.4 Collimation efficiency with various physical apertures. Designed physical aperture sizes correspond to 1.0 of physical aperture ratio.

The value of $70 \%$ physical aperture size is almost the same as the aperture size of primary collimators, so collimation efficiency is much worse. Collimation efficiency improves with wider physical aperture, but it almost saturated beyond the present design value. More large aperture sizes are not so effective as the cost of gap/bore extension. Present designs are reasonable values from the point of collimation.

\subsection{Effect of halo growth rate.}

Because it is not so easy to estimate the halo growth, we assumed the halo growth rate in a simple way. In this way, halo growth rate is dealt with the constant from the summation of overall instabilities and space charge.

In this study, the betatron oscillation was assumed to increase at each element of the synchrotron. We prepared initial particles which are distributed within $216 \pi \mathrm{mm}$.mrad emittance transversely and within $1 \%$ momentum longitudinally. And constant increments in $\mathrm{X}$ and $\mathrm{Y}$ values are added at every elements during tracking. Collimation efficiencies of several constant growth rates are shown in Fig.5.

If constant rate is over $1.15 \mathrm{~mm}$, growth speed is very fast and all particles cannot rotate the synchrotron even only one turn. In the range from $1.15 \mathrm{~mm}$ to $0.15 \mathrm{~mm}$, particles can rotate the ring several turns, and collimation efficiency rise with smaller constant rate. And in this region, collimation efficiency has some extrema. The detail of this property will be investigated from now.
When the constant rate becomes less than $0.15 \mathrm{~mm}$, collimation efficiency reaches about $99 \%$, and this value is same as the result of stable halo collimation.

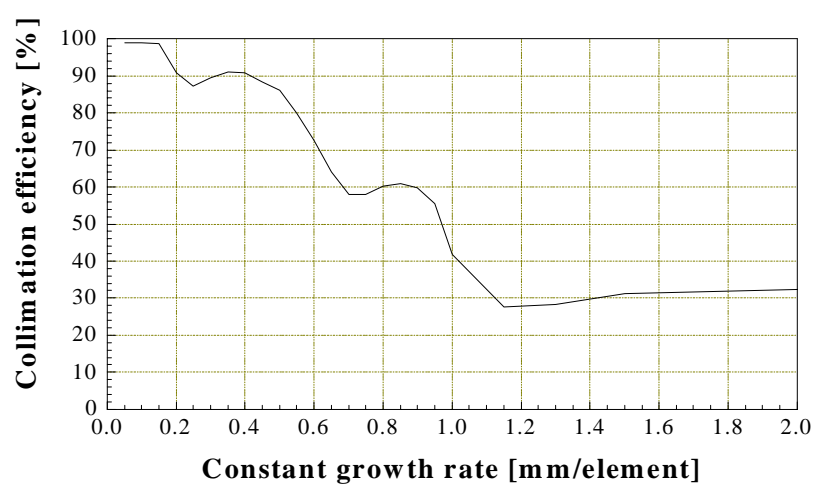

Fig.5 Collimation efficiency dependence on the halo growth rate. The position coordinates of tracking particles are increased by a constant step at every elements.

\section{CONCLUSIONS}

We designed the beam collimation system for the $3 \mathrm{GeV}$ synchrotron and investigated the collimation efficiency for this system. The results are,

1) The collimation system of $3 \mathrm{GeV}$ synchrotron can be applied at two operation points in transverse halo collimation, but collimation efficiency of off momentum particle depends on the operation point.

2) The physical aperture sizes of present designs are reasonable values from view point of collimation efficiency.

3) Collimation efficiency depends on the halo growth rate. This collimation system is effective in the case of the growth rate less than $0.15 \mathrm{~mm}$.

\section{REFERENCES}

[1] Y. Yamazaki, "The present status of the JAERI/KEK Joint Project for High Intensity proton accelerators", in this meeting.

[2] H.Yokomizo et al, "Overview of $3 \mathrm{GeV}$ Rapid Cycle Synchrotron for JAERI-KEK Joint Project", the proceedings of ICANS-XV, Tsukuba, Japan, JAERIConf 2001-002, KEK Proceedings 2000-22, March 2001.

[3] N.Mokhov and W.Chou et al, "Beam Halo and Scraping", the report of the $7^{\text {th }}$ ICFA Mini-workshop, Lake Como, Wisconsin, 1999.

[4] JHP Project Office, "JHF ACCELERATOR DESIGN STUDY REPORT”, KEK Report 97-16, JHF-97-10, March 1998.

[5] T.Trenkler and J.B.Jeanneret, "The principles of two stage betatron and momentum collimation in circular accelerators", CERN-SL/95-03(AP), February 1999.

[6] A.Drozhdin, N.Mokhov, "The STRUCT Program User's Reference Manual”, FNAL, January 2001. 